\title{
From McKeown to Ivor Lewis, the learning curve for thoracic lymphadenectomy over the first 100 robotic esophagectomy cases: a retrospective study
}

\author{
Ze-Guo Zhuo ${ }^{1 \#}$, Gang Li ${ }^{1,2 \#}$, Tie-Niu Song ${ }^{1}$, Gu-Ha Alai ${ }^{1}$ Xu Shen ${ }^{1}$, Yun Wang ${ }^{1}$, Yi-Dan Lin ${ }^{1}$ \\ ${ }^{1}$ Department of Thoracic Surgery, West China Hospital, Sichuan University, Chengdu, China; ${ }^{2}$ Department of Thoracic Surgery, Hospital of \\ Chengdu Office of People's Government of Tibetan Autonomous Region, Chengdu, China \\ Contributions: (I) Conception and design: YD Lin, Y Wang; (II) Administrative support: YD Lin, Y Wang; (III) Provision of study materials or \\ patients: YD Lin, Y Wang; (IV) Collection and assembly of data: ZG Zhuo, G Li, TN Song, GH Alai, X Shen; (V) Data analysis and interpretation: \\ ZG Zhuo, G Li; (VI) Manuscript writing: All authors; (VII) Final approval of manuscript: All authors. \\ "These authors contributed equally to this work. \\ Correspondence to: Yi-Dan Lin; Yun Wang. Department of Thoracic Surgery, West China Hospital, Sichuan University, 37 Guoxue Alley, Chengdu \\ 610041, China. Email: linyidan.academy@foxmail.com; yunwwang@yeah.net.
}

Background: Lymphadenectomy is an essential but challenging part of the surgical treatment for esophageal cancer. However, the previously reported learning curve for robotic esophagectomy primarily focused on only one surgical approach (McKeown or Ivor Lewis). However, both approaches must be mastered by a mature robotic surgical team to deal with different clinical conditions and satisfy patients' needs. This study aimed to show how an experienced esophageal surgical team became proficient in both McKeown and Ivor Lewis robotic esophagectomy.

Methods: A retrospective review of the first 100 cases of robot-assisted minimally invasive esophagectomy (RAMIE) by a single surgical team was performed. The cumulative sum (CUSUM) analysis was used to distinguish the change point during the learning course. A subgroup analysis was performed according to a surgical approach (McKeown or Ivor Lewis) to determine the effect of experience from one surgical approach on learning the other RAMIE technique.

Results: According to the tendency of the CUSUM plot, the learning curve was divided into four phases. The subgroup analysis indicated the decline of the CUSUM plot in the 3rd phase originated from the start of the Ivor Lewis approach. The attending surgeon took 23 cases to achieve a significant improvement in the number of harvested thoracic lymph nodes using the McKeown approach. Regardless of the acquired experience of McKeown RAMIE, it took another 18 cases for the surgical team to achieve significant improvement in harvesting thoracic lymph nodes using the Ivor Lewis approach.

Conclusions: Twenty-three cases were needed for an experienced surgical team to improve thoracic lymphadenectomy results using McKeown RAMIE. There was another learning phase during the transition from McKeown to Ivor Lewis esophagectomy. Importantly, the acquired experience from performing McKeown RAMIE could shorten how long it takes to learn Ivor Lewis RAMIE.

Keywords: Esophageal cancer; lymphadenectomy; robotic surgery; learning curve

Submitted Aug 18, 2020. Accepted for publication Jan 07, 2021.

doi: $10.21037 /$ jtd-20-2862

View this article at: http://dx.doi.org/10.21037/jtd-20-2862 


\section{Introduction}

Esophageal cancer is the $6^{\text {th }}$ leading cause of cancerrelated death worldwide, and surgery is the best choice for patients with resectable disease $(1,2)$. In addition to surgical resection of the tumor, lymphadenectomy is also a crucial part of a successful surgery $(3,4)$. A thorough lymphadenectomy ensures removal of potentially involved lymph nodes and guarantees maximal clearance of the tumor cell (5). Both the number of involved lymph nodes and the number of total harvested lymph nodes are independent predictors of postsurgical survival $(6,7)$. Therefore, special attention should be paid to the quality of lymphadenectomy. According to the $8^{\text {th }}$ edition of the American Joint Committee on Cancer (AJCC) staging criteria, there are 15 thoracic lymph node stations and five abdominal lymph node stations potentially involved in esophageal cancer (8). The National Comprehensive Cancer Network (NCCN) esophageal cancer treatment guidelines recommend that at least 15 lymph nodes be submitted for pathological evaluation to guarantee the accuracy of nodal staging (1).

Considering the importance of harvesting lymph nodes, the learning curve for lymphadenectomy in robotic esophageal surgery has drawn the interest of thoracic surgeons, and a few studies on this topic have been published $(9,10)$. However, thoracic lymphadenectomy, which is the most technically challenging part and involves 15 lymph node stations, has not yet been independently studied. This study aimed to display the learning curve for thoracic lymphadenectomy over the first 100 robotassisted minimally invasive esophagectomy (RAMIE) cases according to the number of harvested thoracic lymph nodes. A subgroup analysis according to surgical approach (McKeown or Ivor Lewis) was also performed to determine the effect of learning one surgical approach on learning the other RAMIE technique. We present the following article in accordance with the MDAR and STROBE reporting checklist (available at http://dx.doi.org/10.21037/jtd-202862).

\section{Methods}

This study was conducted in accordance with the Declaration of Helsinki (as revised in 2013). The Ethics Committee of West China Hospital of Sichuan University approved this study (20190402). Informed consent to undergo robotic surgery without receiving financial support from the national health insurance system was obtained from all patients.

A retrospective review of the first 100 RAMIE cases by a single surgical team in West China Hospital was performed. The surgical team is also experienced in open esophagectomy and conventional thoracoscopic minimal invasive esophagectomy (CMIE). The team started RAMIE in 2016. In the beginning, only the McKeown approach was adopted. Then, based on the acquired experience in the McKeown approach, the surgical team expanded the robotic surgery to the Ivor Lewis technique. The detailed surgical techniques of the two approaches have been described previously $(11,12)$. Cases that were converted to open surgery during RAMIE were excluded from the first 100 cases. CMIE cases performed by the surgical team during the same period was also collected as the control group to monitor surgical quality.

\section{Baseline data collection}

Baseline patient data, including age, gender, body mass index (BMI), preoperative comorbidities, tumor location, date of surgery, and surgical approach, were collected from the electronic medical records system. Postoperative pathologic reports were exported from the electronic pathologic report system. Malignancies were staged according to the eighth edition of the AJCC staging criteria for esophageal cancer (8).

\section{Number of harvested lymph nodes}

In this study, the relevant lymph nodes were divided into two groups: thoracic lymph nodes and abdominal lymph nodes. Although there have been some changes in the classifications of lymph node stations from the $7^{\text {th }}$ edition to $8^{\text {th }}$ edition of the AJCC staging criteria, these changes have no impact on thoracic and abdominal lymph node classifications $(8,13)$. For example, the $3 \mathrm{P}$ station in the $7^{\text {th }}$ edition was renamed as the $8 \mathrm{U}$ station in the $8^{\text {th }}$ edition. However, in both the $7^{\text {th }}$ and $8^{\text {th }}$ editions, it belongs to the thoracic lymph nodes. Nonetheless, the pathologic reports of some early cases in this study were based on the $7^{\text {th }}$ edition. In summary, the thoracic lymph stations included 2L, 2R, 8U, 4L, 4R, 5, 6, 7, 8M, 8Lo, 9L, 9R, 10L, 10R, $15^{\text {th }}$ stations, and the $3 \mathrm{P}, 8 \mathrm{~L}$, and $9^{\text {th }}$ stations in the $7^{\text {th }}$ edition. The abdominal lymph node stations included the $16^{\text {th }}, 17^{\text {th }}, 18^{\text {th }}, 19^{\text {th }}$, and $20^{\text {th }}$ lymph node stations. 
Table 1 The baseline characteristics of the first 100 patients who underwent robot-assisted minimally invasive esophagectomy

\begin{tabular}{|c|c|}
\hline Variables & $\mathrm{N}=100$ \\
\hline Age (year old) & $61.89 \pm 7.71$ \\
\hline \multicolumn{2}{|l|}{ Gender } \\
\hline Male & $84(84 \%)$ \\
\hline Female & $16(16 \%)$ \\
\hline BMI $\left(\mathrm{kg} / \mathrm{m}^{2}\right)$ & $23.10 \pm 3.61$ \\
\hline Diabetes & $5(5 \%)$ \\
\hline Hypertension & $26(26 \%)$ \\
\hline \multicolumn{2}{|l|}{ Tumor location } \\
\hline Upper thorax & $13(13 \%)$ \\
\hline Middle thorax & $50(50 \%)$ \\
\hline Lower thorax & $37(37 \%)$ \\
\hline \multicolumn{2}{|l|}{ Histology } \\
\hline Squamous cell carcinoma & 88 \\
\hline Adenocarcinoma & 6 \\
\hline Other & 6 \\
\hline \multicolumn{2}{|l|}{ Pathologic T stage } \\
\hline Tis & 1 \\
\hline $\mathrm{T} 1$ & 20 \\
\hline $\mathrm{T} 2$ & 24 \\
\hline T3 & 49 \\
\hline $\mathrm{T} 4 \mathrm{a}$ & 6 \\
\hline \multicolumn{2}{|l|}{ Pathologic N stage } \\
\hline No & 52 \\
\hline N1 & 26 \\
\hline N2 & 15 \\
\hline N3 & 7 \\
\hline \multicolumn{2}{|l|}{ TNM stage } \\
\hline 0 & 1 \\
\hline I & 14 \\
\hline II & 42 \\
\hline III & 34 \\
\hline IVa & 9 \\
\hline \multicolumn{2}{|l|}{ Surgical approach } \\
\hline McKeown & 69 \\
\hline Ivor Lewis & 31 \\
\hline Number of RCLN-LN & $2.19 \pm 2.31$ \\
\hline Number of Thoracic LN & $9.97 \pm 4.78$ \\
\hline Number of Abdomen LN & $10.66 \pm 6.87$ \\
\hline Number of Total LN & $20.63 \pm 8.30$ \\
\hline
\end{tabular}

BMI, body mass index; LN, lymph node; RLN-LN, recurrent laryngeal nerve lymph node.

\section{Statistical analysis}

The cumulative sum (CUSUM) analysis is one of the most well-known monitoring methods for sequential data (14). CUSUM values were calculated in Excel 2010 (Microsoft, Redmond, WA, USA) according to the following formula: CUSUM $=\sum_{i}^{100}(x i-u)$, where $x i$ stands for the true value of the $\mathrm{i}^{\text {th }}$ case and $u$ stands for the mean value of the examined variable. Changes in the CUSUM value reflect the running total of data deviation from the mean value of the group. If the actual measured value is above the group's mean value, then CUSUM values will increase. Thus, we can observe the changing tendency of sequential data by the changing CUSUM value. Points with the largest peaks in the CUSUM curve were identified as the change points of the learning curve. The significance of the change points was further tested by comparing the true value before and after the point. Subgroup analysis was performed according to surgical approach (McKeown or Ivor Lewis) to see the individual learning curves of the two surgical approaches.

SPSS 22.0 software (IBM, Armonk, NY, USA) was used for all statistical analyses and to draw the CUSUM learning curves. Student's $t$-test or one-way ANOVA were applied to compare the integrated parameters, while the Chi-squared test or Fisher's exact test were applied to dichotomous data. $\mathrm{P}$ values $<0.05$ were considered statistically significant.

\section{Results}

The surgical team accomplished their $100^{\text {th }}$ case of robotic esophagectomy in February 2018. Two cases were converted to open surgery during the period, one due to severe pleural adhesion and another due to large mixed lymph nodes invading the celiac trunk. Both were excluded from the first 100 cases. Table 1 shows baseline characteristics of the first 100 patients who underwent RAMIE. The mean age of the patients was 61.89-years-old, with a mean BMI of $23.10 \mathrm{~kg} / \mathrm{m}^{2}$. The number of male patients was much greater than the number of female patients (86 versus 14 , respectively). Twenty-six patients had a history of hypertension, while five had a history of diabetes. Squamous cell carcinoma (88\%) was the most common histologic type. Fifty patients had a middle thoracic tumor, 37 had a lower thoracic tumor, and the remaining 13 had tumors in the upper thorax. Regarding surgical approach, the McKeown approach was adopted in 69 cases, while the Ivor Lewis approach was used in 31 . The mean numbers of harvested recurrent laryngeal nerve lymph nodes, thoracic lymph 


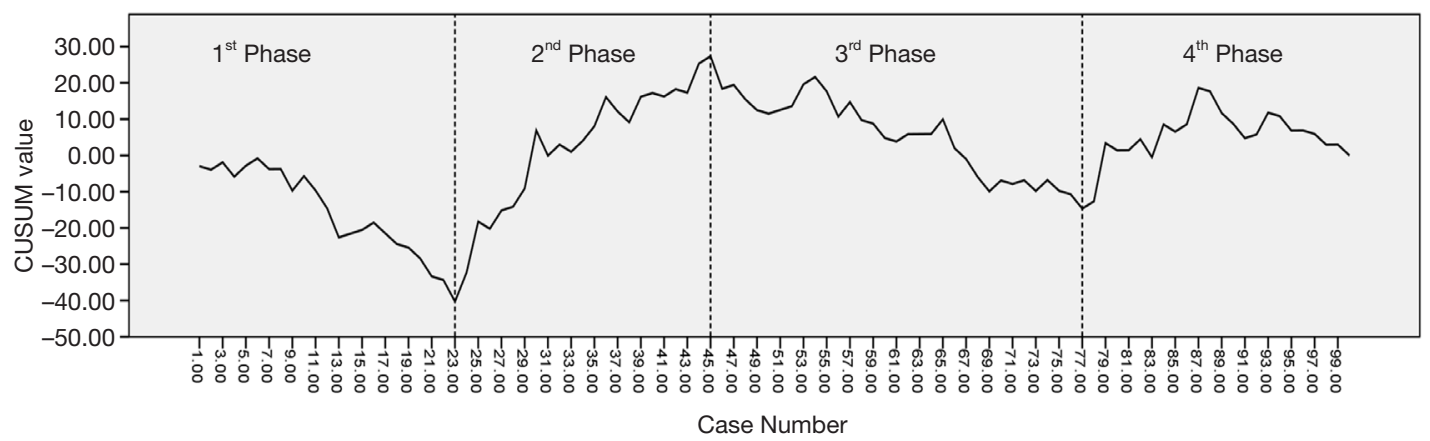

Figure 1 The CUSUM plot of the number of harvested thoracic lymph nodes in the first 100 cases of robot-assisted minimally invasive esophagectomy from a single surgical team. By visual assessment on the CUSUM plot, the learning curve was divided into 4 phases by three turning points (the $23^{\text {rd }}$ case, the $45^{\text {th }}$ case, the $77^{\text {th }}$ case), after which the tendency of the CUSUM plot changed. The first phase consisted of the first 23 cases. In this phase, the CUSUM plot trended downward, which meant the number of harvested lymph nodes in most cases was beneath the mean value of the 100 cases. After the $23^{\text {rd }}$ case, the CUSUM plot trended to rise up and reached a peak when it came to the $45^{\text {th }}$ case. So the $24^{\text {th }}$ to $45^{\text {th }}$ made the second phase. Then, another decline occurred between the $46^{\text {th }}$ to $77^{\text {th }}$ cases. It was the $3^{\text {rd }}$ phase. After the $3^{\text {rd }}$ phase, the CUSUM value waved around zero, and the CUSUM plot becomes steady. It made the fourth phase. CUSUM, cumulative sum.

nodes, and abdominal lymph nodes were 2.19, 9.97, and 10.66 , respectively. Lymph node metastasis was detected in 48 patients.

\section{The learning curve for lymphadenectomy in the first 100 robotic esophagectomies}

Visual assessment of the CUSUM plot of the first 100 robotic esophagectomies (Figure 1) showed three turning points, after which the trend of the CUSUM plot changed. These three turning points divided the learning curve for thoracic lymphadenectomy into four phases. The first phase consisted of the first 23 cases. In this phase, the CUSUM plot trended downward, which meant the number of harvested lymph nodes in most cases was beneath the mean value of the 100 cases. After the $23^{\text {rd }}$ case, the CUSUM plot had an increasing trend that peaked by the $45^{\text {th }}$ case. So the $24^{\text {th }}$ to $45^{\text {th }}$ cases comprised the second phase. Then, another decline occurred between the $46^{\text {th }}$ to $77^{\text {th }}$ cases, which was the $3^{\text {rd }}$ phase. After the $3^{\text {rd }}$ phase, the CUSUM value wavered around zero, and the CUSUM plot became steady. This was the fourth phase.

The comparison of patients' baseline characteristics and the number of harvested lymph nodes among the four phases are shown in Table 2. The mean number of harvested abdominal lymph nodes ranged from 10.06 to 11.35 , and it was comparable among the four phases. However, a statistically significant difference was detected in the number of harvested thoracic lymph nodes. The mean number of harvested thoracic lymph nodes in the $2^{\text {nd }}$ phase was 13.05 and was 10.61 in the $4^{\text {th }}$ phase. On the contrary, the $1^{\text {st }}$ and $3^{\text {rd }}$ phases had much smaller mean numbers of harvested thoracic lymph nodes, 8.22 and 8.66, respectively. Due to the steady number of harvested abdominal lymph nodes, the differences among the four phases in the total number of harvested lymph nodes was consistent with the thoracic lymph nodes. The baseline characteristics, including age, gender, BMI, comorbidities, tumor location, and disease stage, were comparable among the four phases. However, the four phases showed significant differences in the surgical approaches. All cases in the first phase underwent the McKeown approach. In the second phase, the Ivor Lewis approach was applied for the first time. But only three patients underwent the new approach. However, the number of Ivor Lewis cases was almost equal to those that underwent the McKeown approach in the third phase, and it exceeded the McKeown approach in the fourth phase.

\section{Lymphadenectomy quality in the four learning phases}

During the same period, the surgical team performed 49 CMIE procedures in addition to the 100 RAMIE cases (Table S1). The mean number of total harvested lymph nodes was 17.86 in the 49 patients who underwent CMIE. More lymph nodes were harvested from all four RAMIE learning phases than CMIE. Moreover, the difference between the $2^{\text {nd }}$ and $4^{\text {th }}$ phases was statistically significant. The number of harvested recurrent laryngeal nerve lymph 
Table 2 Comparison of patients' characteristics and the number of harvested lymph nodes among the four learning phases

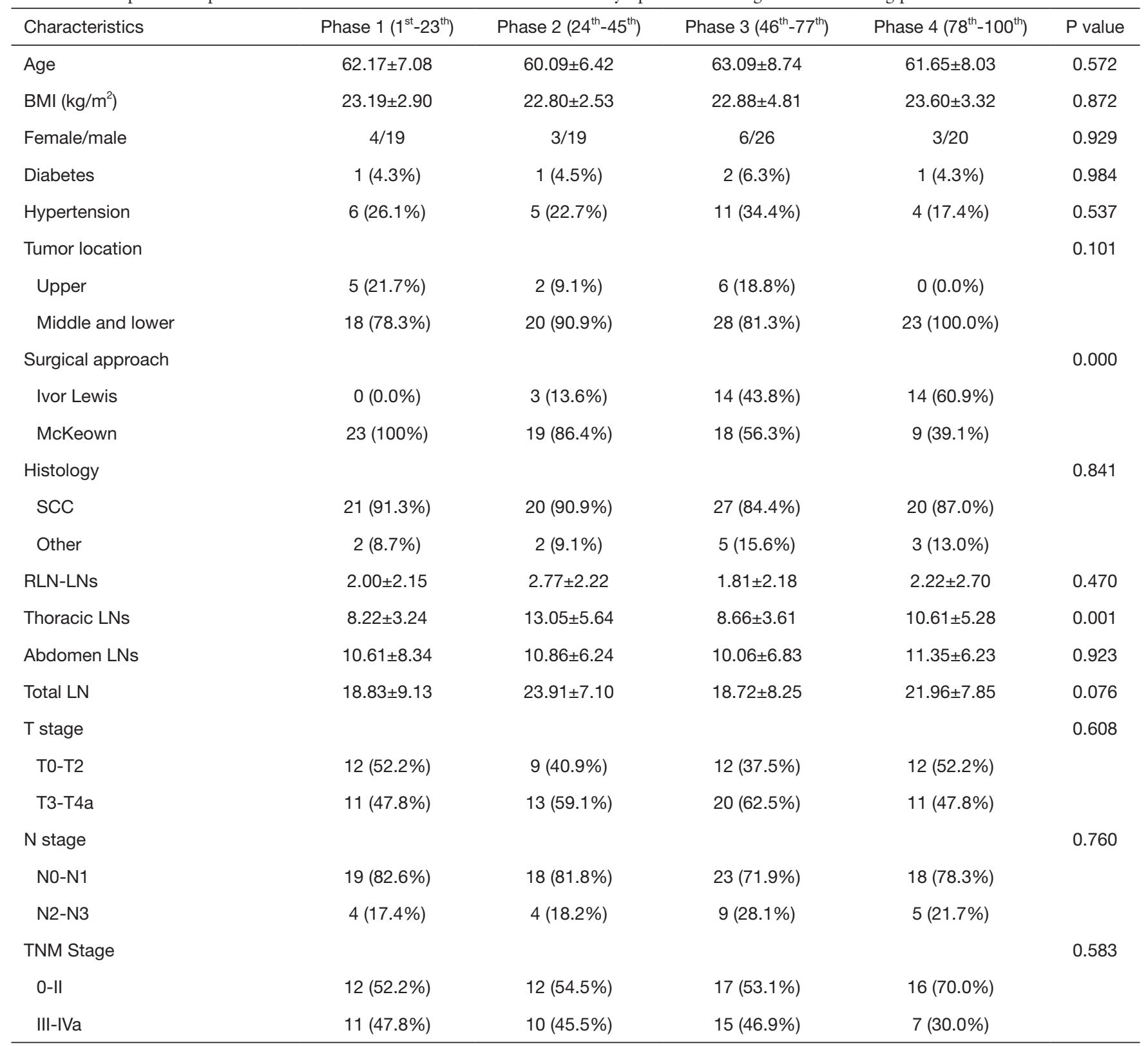

BMI, body mass index; SCC, squamous cell carcinoma; LN, lymph node; RLN-LN, recurrent laryngeal nerve lymph node.

nodes was comparable between CMIE and the four RAMIE learning phases. Therefore, the lymphadenectomy quality of RAMIE was satisfactory.

\section{Lymphadenectomy learning curves according to surgical approach}

A subgroup analysis was performed according to surgical approach to determine if the change in surgical approach led to the decline in the CUSUM plot in the $3^{\text {rd }}$ phase. Among the 32 cases in the $3^{\text {rd }}$ phase, 14 underwent the Ivor Lewis approach, while the remaining 18 cases adopted the McKeown approach. The mean numbers of harvested lymph nodes were 7.14 and 9.83 in the Ivor Lewis and McKeown approaches, respectively, which was a statistically significant difference $(\mathrm{P}=0.034)$. So the decrease in the 

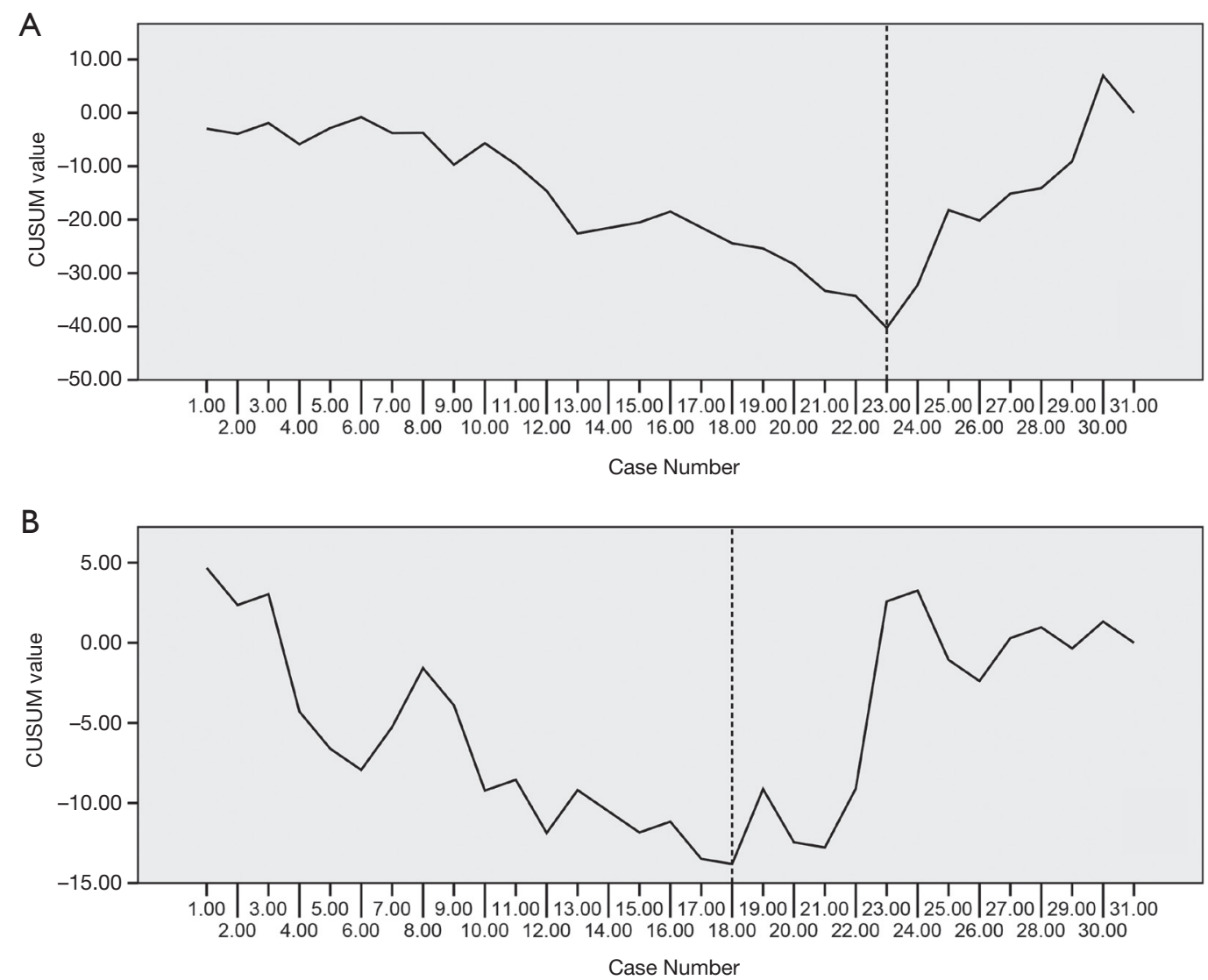

Figure 2 The CUSUM plot of the number of harvested thoracic lymph nodes in ro-bot-assisted McKeown and Ivor Lewis esophagectomy separately. (A) The CUSUM plot of the number of harvested thoracic lymph nodes in the first 31 cases of robot-assisted McKeown esophagectomy. The tendency of the CUSUM plot changed from downward to upward in the 23rd case. It indicated that it took 23 cases for the surgeons to accomplish the initial exploration of the harvest of the thoracic lymph nodes in the McKeown approach. (B) The CUSUM plot of the number of harvested thoracic lymph nodes in the first 31 cases of robot-assisted Ivor Lewis esophagectomy. The CUSUM plot tended to downward at the beginning, and it reached the lowest point in the 18th case. After that, the CUSUM plot started ascending. It indicated that on the basis of the robot-assisted McKeown lymphade-nectomy, the exploration of the thoracic lymphadenectomy in the Ivor Lewis approach took another 18 cases. CUSUM, cumulative sum.

CUSUM learning curve in the $3^{\text {rd }}$ phase resulted from the increasing application of the Ivor Lewis approach. Although the Ivor Lewis approach was launched on the basis of the accumulated experience from the McKeown approach, due to the change in body position and port design, the lymphadenectomy still involved a learning curve. The independent learning curves for dissecting thoracic lymph nodes using the Ivor Lewis and McKeown approaches are shown in Figure 2. It took 23 cases for the surgeons to accomplish the initial increase in the harvest of thoracic lymph nodes using the McKeown approach (Figure 2A). However, based on knowledge from the robotic McKeown lymphadenectomy, increasing the yield from lymphadenectomy using the Ivor Lewis approach only took 18 cases (Figure $2 B$ ).

\section{Comparison between McKeown RAMIE and Ivor Lewis RAMIE}

We next compared the two surgical approaches to determine if they were equivalent with regard to lymphadenectomy (Table 3). The mean number of total harvested lymph nodes in the learning phase of McKeown RAMIE ( $1^{\text {st }}$ to $23^{\text {rd }}$ cases) was 18.83 , and that of the learning phase of Ivor Lewis RAMIE ( $1^{\text {st }}$ to $18^{\text {th }}$ cases) was also 18.83 . Both surgical approaches improved the number 
Table 3 Comparison of lymphadenectomy between McKeown and Ivor Lewis RAMIE in the learning and commanding phase

\begin{tabular}{|c|c|c|c|c|c|c|}
\hline Characteristics & \multicolumn{3}{|c|}{ Learning phase of } & \multicolumn{3}{|c|}{ Commanding phase of } \\
\hline Age & $62.17 \pm 7.08$ & $63.44 \pm 8.77$ & 0.610 & $61.67 \pm 7.38$ & $60.00 \pm 8.77$ & 0.491 \\
\hline BMI $\left(\mathrm{kg} / \mathrm{m}^{2}\right)$ & $23.19 \pm 2.90$ & $22.80 \pm 2.53$ & 0.483 & $22.62 \pm 3.05$ & $23.04 \pm 2.32$ & 0.643 \\
\hline Female/male & $4 / 19$ & $3 / 15$ & 1.000 & $8 / 38$ & $1 / 12$ & 0.668 \\
\hline Hypertension & $6(26.1 \%)$ & $5(27.8 \%)$ & 0.903 & $12(26.1 \%)$ & $3(23.1 \%)$ & 1.000 \\
\hline Tumor location & & & 0.056 & & & 0.180 \\
\hline Upper & $5(21.7 \%)$ & $0(0.0 \%)$ & & $8(17.4 \%)$ & $0(0.0 \%)$ & \\
\hline Middle and lower & $18(78.3 \%)$ & $18(100.0 \%)$ & & $38(82.6 \%)$ & $13(100.0 \%)$ & \\
\hline Abdomen LNs & $10.61 \pm 8.34$ & $11.28 \pm 6.22$ & 0.778 & $9.98 \pm 6.32$ & $12.31 \pm 7.22$ & 0.260 \\
\hline Total LN & $18.83 \pm 9.13$ & $18.83 \pm 7.29$ & 0.998 & $21.93 \pm 8.47$ & $21.69 \pm 7.28$ & 0.926 \\
\hline T stage & & & 0.063 & & & 0.701 \\
\hline T0-T2 & $12(52.2 \%)$ & $4(22.2 \%)$ & & $22(47.8 \%)$ & $7(53.8 \%)$ & \\
\hline T3-T4a & $11(47.8 \%)$ & $14(77.8 \%)$ & & $24(52.2 \%)$ & $6(46.2 \%)$ & \\
\hline $\mathrm{N}$ stage & & & 0.087 & & & 0.019 \\
\hline No-N1 & $19(82.6 \%)$ & $10(55.6 \%)$ & & $41(89.1 \%)$ & $8(61.5 \%)$ & \\
\hline N2-N3 & $4(17.4 \%)$ & 8 (44.4\%) & & 5 (10.9\%) & 5 (38.5\%) & \\
\hline
\end{tabular}

BMI, body mass index; LN, lymph node; RLN-LN, recurrent laryngeal nerve lymph node.

of total harvested lymph nodes after the learning phase. The mean number of total harvested lymph nodes was 21.93 in the commanding phase of McKeown RAMIE $\left(24^{\text {th }}\right.$ to $69^{\text {th }}$ cases), while it was 21.69 in the commanding phase of Ivor Lewis RAMIE ( $19^{\text {th }}$ to $31^{\text {st }}$ cases). The number of total harvested lymph nodes was comparable between the two surgical approaches in the learning and commanding phases. However, the McKeown approach harvested significantly more recurrent laryngeal nerve lymph nodes than the Ivor Lewis approach. As Table 3 shows, the McKeown approach had more patients with tumors in the upper third esophagus. In consideration of this, we think it was the tumor location but not the surgical approach that led to more recurrent laryngeal nerve lymph nodes in McKeown cases.

\section{Discussion}

To the best of our knowledge, this is the first study to record the actual learning course of a single surgical team from the initial start of McKeown RAMIE to mastery of both McKeown and Ivor Lewis RAMIE. Our CUSUM analysis indicated that the experienced esophageal surgical team needed to perform 23 cases of McKeown RAMIE before achieving significant improvements in the harvest of thoracic lymph nodes. Despite their thoracic lymphadenectomy experience using the McKeown approach, the transition from McKeown RAMIE to Ivor Lewis RAMIE still involved a learning curve. It took another 18 cases for the surgical team to accomplish the transition. RAMIE provides the same lymphadenectomy 
quality as CMIE but could be better after the learning period.

Strengths of robot-assisted surgical systems are threedimensional vision, articulated instruments, dexterity, and enhanced ergonomics $(15,16)$. These allow surgeons to have a better view of the surgical field and more flexible surgical instruments. Robot-assisted surgical systems have natural advantages for performing lymphadenectomies compared with conventional thoracoscopic surgical systems. Oshikiri et al. reported that it took 33 cases to improve the number of total harvested lymph nodes for a team learning CMIE (17). Park et al. reported an increased number of harvested lymph nodes in the $28^{\text {th }}$ case using RAMIE (10). In our study, a significant improvement in the number of harvested thoracic lymph nodes was achieved after the $23^{\text {rd }}$ case. It looks fewer cases to learn RAMIE than to learn CMIE. The shorter learning curve of lymphadenectomy using RAMIE may be due in part to the robotic surgical system itself and previous CMIE experience. To date, no study has reported the learning curve from open esophagectomy directly to RAMIE. So, we are currently unable to comment on the effect of previous CMIE experience on learning RAMIE.

The previously reported learning curve of RAMIE primarily focused on only one surgical approach (McKeown or Ivor Lewis) (18). The McKeown approach is necessary for patients with upper esophageal tumors, especially those above the carina (19). Both the McKeown and Ivor Lewis approaches are optimal for middle and lower thoracic esophageal cancer. However, the Ivor Lewis technique is less traumatic, and Yang et al. report better quality of life in patients who underwent Ivor Lewis esophagectomy (20). Thus, patients seeking less surgical trauma and higher quality of life will prefer the Ivor Lewis approach. For a mature robotic surgical team to be able to deal with different clinical conditions and satisfy all patients' needs, both approaches must be mastered. Therefore, while the team is learning RAMIE, experiences with both surgical approaches is imperative. A multicenter study demonstrated that there is still a learning curve for surgeons with previous experience in McKeown CMIE to master the Ivor Lewis CMIE (21). In our study, the Ivor Lewis approach was implemented in the $2^{\text {nd }}$ phase and was widely launched in the $3^{\text {rd }}$ phase. A decline in the CUSUM plot occurred along with the broad application of the Ivor Lewis approach in the $3^{\text {rd }}$ phase. We concluded the transition from McKeown to Ivor Lewis RAMIE required another learning phase. So, a subgroup analysis according to surgical approach was performed. The results showed it took another 18 cases for the surgeons to become proficient in thoracic lymphadenectomy using the Ivor Lewis approach. Compared with the initial learning phase for McKeown RAMIE, the following learning phase for Ivor Lewis RAMIE took fewer cases before the team became proficient at thoracic lymph node dissection.

We also compared RAMIE and CMIE to monitor the surgical quality of RAMIE. The mean number of total harvested thoracic lymph nodes in the $1^{\text {st }}$ and $3^{\text {rd }}$ phases were comparable to CMIE. Moreover, RAMIE harvested significantly more lymph nodes in the $2^{\text {nd }}$ and $4^{\text {th }}$ phases than CMIE approaches. So, the lymphadenectomy quality of the RAMIE approach was at equal to the CMIE approach, and could be better after the learning period.

There were some limitations to this retrospective study. First, this study focused on lymphadenectomy learning curves, and the learning phases were divided according to CUSUM analysis of the number of harvested thoracic lymph nodes. Other short-term outcomes, such as surgery time and postoperative complications, were not included in the analysis. So, this analysis only reflects the learning course of the lymphadenectomy, especially thoracic lymphadenectomy. Second, because of its retrospective nature, we were unable to collect the time cost of the lymphadenectomy procedure. So, we were also unable to further study improvements in the time needed for lymphadenectomy procedures.

The overall rate of neoadjuvant therapy in China is low, and in this study, only three of the 100 patients received neoadjuvant therapy. Most of our patients were from rural areas, and they had an urgent need to solve the dysphagia caused by the tumor. Thus, they had a strong preference to undergo surgery as soon as possible. Moreover, the patients were afraid of disease progression during neoadjuvant therapy that could cost them the chance for surgery. Therefore, almost all patients were unwilling to receive neoadjuvant therapy. These were the reasons why the neoadjuvant therapy rate was quite low in our patients.

In summary, our study showed the learning curve for an experienced surgical team while they mastered the McKeown and Ivor Lewis RAMIE techniques, and then analyzed interactions between learning the two surgical approaches. These lessons can be helpful and instructional for other teams learning robotic esophageal surgery. For example, surgeons who are learning or planning to learn robotic esophageal surgery techniques could know how long it normally takes before they become proficient in the 
surgical approach and how the changes happened during the learning process.

\section{Acknowledgments}

We would like to thank Mr. Shi-De Wu from the High School Attached to Northeast Normal University for his linguistic assistance to this manuscript.

Funding: This work was supported by the National Natural Science Foundation of China (grant number: 81672291, 31071210 to YD Lin).

\section{Footnote}

Reporting Checklist: The authors have completed the MDAR and STROBE reporting checklist. Available at http://dx.doi. org/10.21037/jtd-20-2862

Data Sharing Statement: Available at http://dx.doi. org/10.21037/jtd-20-2862

Conflicts of Interest: All authors have completed the ICMJE uniform disclosure form (available at http://dx.doi. org/10.21037/jtd-20-2862). The authors have no conflicts of interest to declare.

Ethical Statement: The authors are accountable for all aspects of the work in ensuring that questions related to the accuracy or integrity of any part of the work are appropriately investigated and resolved. The study was conducted in accordance with the Declaration of Helsinki (as revised in 2013). The study was approved by the ethics committee of West China Hospital, Sichuan University (No. 20190402). Informed consent to undergo robotic surgery without receiving financial support from the national health insurance system was obtained from all patients.

Open Access Statement: This is an Open Access article distributed in accordance with the Creative Commons Attribution-NonCommercial-NoDerivs 4.0 International License (CC BY-NC-ND 4.0), which permits the noncommercial replication and distribution of the article with the strict proviso that no changes or edits are made and the original work is properly cited (including links to both the formal publication through the relevant DOI and the license). See: https://creativecommons.org/licenses/by-nc-nd/4.0/.

\section{References}

1. Ajani JA, D'Amico TA, Bentrem DJ, et al. Esophageal and Esophagogastric Junc-tion Cancers, Version 2.2019, NCCN Clinical Practice Guidelines in Oncology. J Natl Compr Canc Netw 2019;17:855-83.

2. Martin-Richard M, Diaz Beveridge R, Arrazubi V, et al. SEOM Clinical Guideline for the diagnosis and treatment of esophageal cancer (2016). Clin Transl Oncol 2016;18:1179-86.

3. Zhan C, Shi Y, Jiang W, et al. How many lymph nodes should be dissected in esophagectomy with or without neoadjuvant therapy to get accurate staging? Dis Esophagus 2020;33:1-8.

4. Dudash MJ, Slipak S, Dove J, et al. Lymph Node Harvest as a Measure of Quality and Effect on Overall Survival in Esophageal Cancer: A National Cancer Database Assessment. Am Surg 2019;85:201-5.

5. Tachimori Y. Pattern of lymph node metastases of squamous cell esophageal cancer based on the anatomical lymphatic drainage system: efficacy of lymph node dissection according to tumor location. J Thorac Dis 2017;9:S724-30.

6. Peyre CG, Hagen JA, DeMeester SR, et al. The number of lymph nodes removed predicts survival in esophageal cancer: an international study on the impact of extent of surgical resection. Ann Surg 2008;248:549-56.

7. Peyre CG, Hagen JA, DeMeester SR, et al. Predicting systemic disease in patients with esophageal cancer after esophagectomy: a multinational study on the significance of the number of involved lymph nodes. Ann Surg 2008;248:979-85.

8. Rice TW, Ishwaran H, Ferguson MK, et al. Cancer of the Esophagus and Esophagogastric Junction: An Eighth Edition Staging Primer. J Thorac Oncol 2017;12:36-42.

9. Park SY, Kim DJ, Kang DR, et al. Learning curve for robotic esophagectomy and dissection of bilateral recurrent laryngeal nerve nodes for esophageal cancer. Dis Esophagus 2017;30:1-9.

10. Park S, Hyun K, Lee HJ, et al. A study of the learning curve for robotic oesophagectomy for oesophageal cancer. Eur J Cardiothorac Surg 2018;53:862-70.

11. Wang WP, Chen LQ, Zhang HL, et al. Modified Intrathoracic Esophagogastrostomy with Minimally Invasive Robot-Assisted Ivor-Lewis Esophagectomy for Cancer. Dig Surg 2019;36:218-25. 
12. Zhang H, Chen L, Wang Z, et al. The Learning Curve for Robotic McKeown Esophagectomy in Patients With Esophageal Cancer. Ann Thorac Surg 2018;105:1024-30.

13. Rice TW, Blackstone EH, Rusch VW. 7th edition of the AJCC Cancer Staging Manual: esophagus and esophagogastric junction. Ann Surg Oncol 2010;17:1721-4.

14. Bolsin S, Colson M. The use of the Cusum technique in the assessment of trainee competence in new procedures. Int J Qual Health Care 2000;12:433-8.

15. Wee JO, Bravo-Iniguez CE, Jaklitsch MT. Early Experience of Robot-Assisted Esophagectomy With Circular End-to-End Stapled Anastomosis. Ann Thorac Surg 2016;102:253-9.

16. van der Sluis PC, van der Horst S, May AM, et al. Robot-assisted Minimally Invasive Thoracolaparoscopic Esophagectomy Versus Open Transthoracic Esophagectomy for Resectable Esophageal Cancer: A Randomized Controlled Trial. Ann Surg 2019;269:621-30.

17. Oshikiri T, Yasuda T, Hasegawa H, et al. Short-

Cite this article as: Zhuo ZG, Li G, Song TN, Alai GH, Shen X, Wang Y, Lin YD. From McKeown to Ivor Lewis, the learning curve for thoracic lymphadenectomy over the first 100 robotic esophagectomy cases: a retrospective study. J Thorac Dis 202 1;13(3):1543-1552. doi: 10.21037/jtd-20-2862 term outcomes and one surgeon's learning curve for thoracoscopic esophagectomy performed with the patient in the prone position. Surg Today 2017;47:313-9.

18. Claassen L, van Workum F, Rosman C. Learning curve and postoperative outcomes of minimally invasive esophagectomy. J Thorac Dis 2019;11:S777-85.

19. van Workum F, Berkelmans GH, Klarenbeek BR, et al. McKeown or Ivor Lewis totally minimally invasive esophagectomy for cancer of the esophagus and gastroesophageal junction: systematic review and metaanalysis. J Thorac Dis 2017;9:S826-33.

20. Yang YS, Shang QX, Yuan Y, et al. Comparison of Longterm Quality of Life in Patients with Esophageal Cancer after Ivor-Lewis, Mckeown, or Sweet Esophagectomy. J Gastrointest Surg 2019;23:225-31.

21. van Workum F, Stenstra M, Berkelmans GHK, et al. Learning Curve and Associated Morbidity of Minimally Invasive Esophagectomy: A Retrospective Multicenter Study. Ann Surg 2019;269:88-94. 


\section{Supplementary}

Table S1 Comparison of the lymphadenectomy quality between conventional MIE and RAMIE during four learning phases

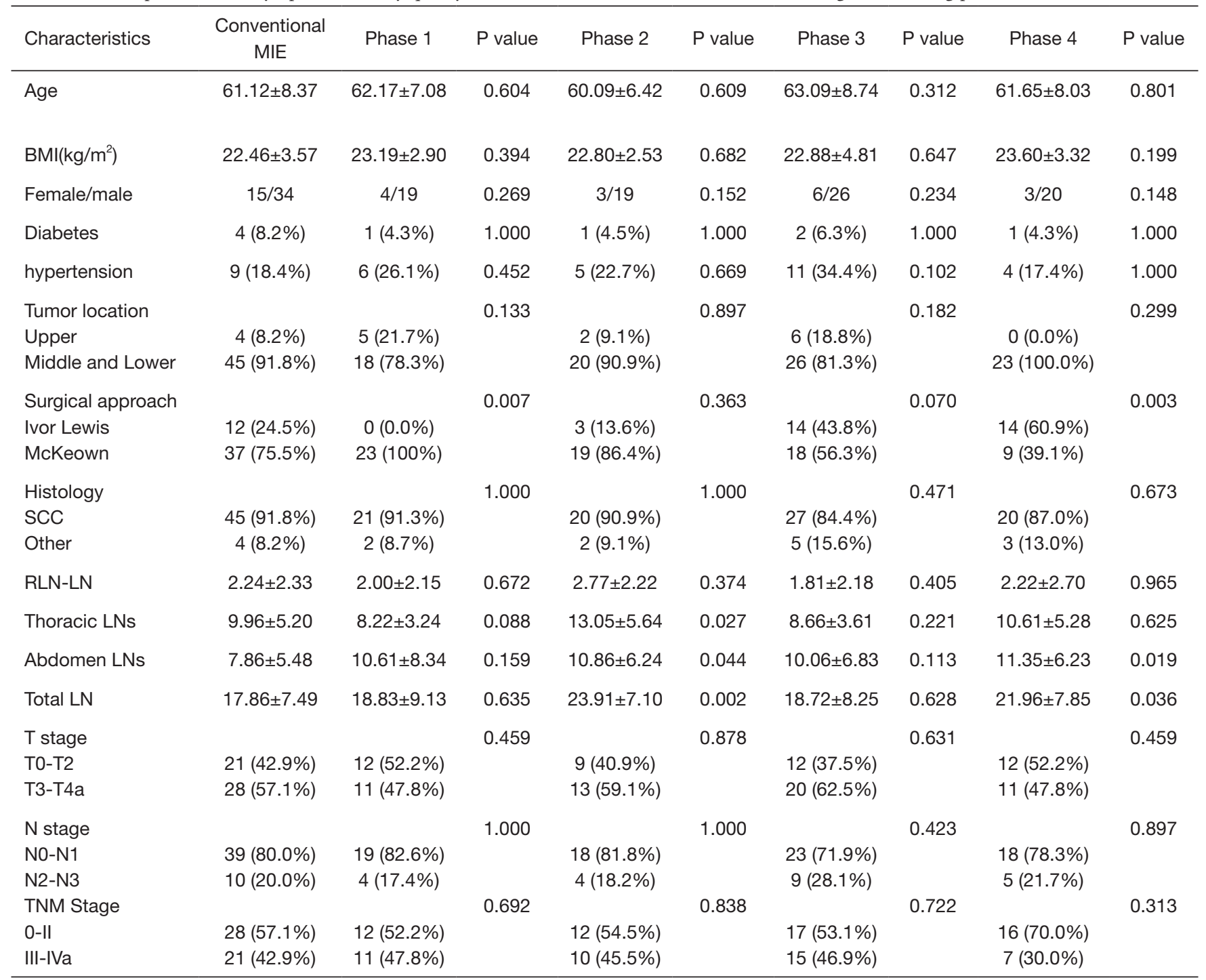

Note: $\mathrm{BMI}=$ body mass index, $\mathrm{SCC}=$ squamous cell carcinoma, $\mathrm{LN}=$ lymph node, $\mathrm{MIE}=$ minimal invasive esophagectomy, $\mathrm{RAMIE}=$ robotassisted minimal invasive esophagectomy, RLN-LN= recurrent laryngeal nerve lymph node 